Pacific Journal of Mathematics

SINGULARITY OF GAUSSIAN MEASURES IN FUNCTION
SPACES WITH FACTORABLE COVARIANCE FUNCTIONS 


\title{
SINGULARITY OF GAUSSIAN MEASURES IN FUNCTION SPACES WITH FACTORABLE COVARIANCE FUNCTIONS
}

\section{J. YEH}

\begin{abstract}
Singularity of Gaussian measures $\mu_{1}$ and $\mu_{2}$ on the function space $R^{\prime \prime}$ of real valued functions $x(t)$ on an arbitrary interval $D$ with factorable covariance functions $r_{i}(s, t)$, i.e., $r_{i}(s, t)=$ $u_{i}(s) v_{i}(t)$ for $s \leq t$ and $r_{i}(s, t)=v_{i}(s) u_{i}(t)$ for $s \geq t, i=1,2$, is treated. Local conditions on the factor functions $u_{\imath}(t)$ and $v_{\imath}(t)$ which insure the singularity of $\mu_{1}$ and $\mu_{2}$ are given.
\end{abstract}

Consider the measurable space $\left(R^{D}, \mathfrak{F}\right)$ where $R^{D}$ is the space of all real valued functions $x(t)$ on a fixed but unspecified interval $D$ of the real line and $\widetilde{\mho}$ is the smallest $\sigma$-field of subsets of $R^{D}$ with respect to which all real valued functions $Y(t, x)=x(t)$ defined on $R^{D}$ with parameter $t \in D$ are measurable. A probability measure $\mu$ on $\left(R^{D}, \mathfrak{F}\right)$ is called a Gaussian measure on the function space $R^{D}$ if the stochastic process $Y(t, x)=x(t)$ on the probability space $\left(R^{D}, \mathfrak{F}, \mu\right)$ with the domain of definition $D$ is a Gaussian process. From the viewpoint of stochastic processes if $X(t, \omega)$ is a stochastic process on an arbitrary probability space $(\Omega, \mathfrak{B}, P)$ with the domain of definition $D$ then a probability measure $\mu_{X}$ is induced on the measurable space $\left(R^{D}, \mathfrak{F}\right)$ by embedding the sample functions $X(\cdot, \omega), \omega \in \Omega$, in $R^{D}$. The stochastic process $Y(t, x)=x(t)$ defined on the probability space $\left(R^{D}, \mathfrak{F}, \mu_{X}\right)$ with the domain of definition $D$ is equivalent to the original process $X(t, \omega)$ so that if $X(t, \omega)$ is a Gaussian process so is $Y(t, x)$. Thus a Gaussian measure on $\left(R^{D}, \mathfrak{F}\right)$ can be defined equivalently as the probability measure $\mu_{X}$ induced on $\left(R^{D}, \mathfrak{F}\right)$ by a Gaussian process $X(t, \omega)$.

J. Feldman [3] and J. Hájek [4], [5] showed independently that any two Gaussian measures are either equivalent or singular. In [7] we applied Hájek's criterion for equivalence or singularity to investigate the singularity of Gaussian measures induced by Brownian motion processes with nonstationary increments. In the present paper we consider the singularity of Gaussian measures $\mu_{1}$ and $\mu_{2}$ on $\left(R^{D}, \mathfrak{F}\right)$ for which the covariance functions $r_{i}(s, t)$ of the stochastic process $Y(t, x)=x(t)$ are factorable. Our main result is the following theorem

THEOREM. Let $\mu_{1}$ and $\mu_{2}$ be Gaussian measures on $\left(R^{D}, \mathfrak{F}\right)$ with zero mean functions and factorable covariance functions $r_{i}(s, t), i=$ 1,2 , given by

$$
r_{i}(s, t)= \begin{cases}u_{i}(s) v_{i}(t) & s \leqq t, s, t \in D, i=1,2 \\ v_{i}(s) u_{i}(t) & s \geqq t, s, t \in D, i=1,2\end{cases}
$$


where $u_{i}(t)$ and $v_{i}(t)$ are nonnegative functions on $D$ satisfying

$$
u_{i}\left(t^{\prime \prime}\right) v_{i}\left(t^{\prime}\right)-u_{i}\left(t^{\prime}\right) v_{i}\left(t^{\prime \prime}\right) \geqq 0 \quad t^{\prime}, t^{\prime \prime} \in D, t^{\prime}<t^{\prime \prime}, i=1,2 .
$$

If there exists $t_{0} \in D$ such that $v_{i}(t)>0$ and $u_{i}(t)\left[v_{i}(t)\right]^{-1}$ are strictly increasing on $\left(t_{0}, t_{0}+\delta\right)$ for some $\delta>0$, the right derivatives $D^{+} u_{i}\left(t_{0}\right)$ and $D^{+} v_{i}\left(t_{0}\right)$ of $u_{i}(t)$ and $v_{i}(t)$ at $t_{0}$ exist and

$$
\begin{array}{ll}
u_{i}\left(t_{0}\right)=0, D^{+} u_{i}\left(t_{0}\right)=\lambda_{i}>0, & i=1,2 \\
v_{i}\left(t_{0}\right)=r_{i}>0, & i=1,2
\end{array}
$$

then the condition

$$
\lambda_{1} r_{1} \neq \lambda_{2} r_{2}
$$

implies the singularity of $\mu_{1}$ and $\mu_{2}$.

We remark that the above theorem can also be stated in terms of the left derivatives of $u_{i}(t)$ and $v_{i}(t)$. When $v_{i}(t)$ are positive on $D$ the condition (1.2) is equivalent to the condition that $u_{i}(t)\left[v_{i}(t)\right]^{-1}$ be nondecreasing on $D$. For a symmetric function $r(s, t), s, t \in D$, defined as in (1.1) by means of two nonnegative functions $u(t)$ and $v(t)$ on $D$ to be the covariance function of a Gaussian process it is necessary and sufficient that for any $t_{1}, \cdots, t_{n} \in D, t_{1}<\cdots<t_{n}$, the $n \times n$ matrix $\left[r\left(t_{k}, t_{l}\right), k, l=1,2, \cdots, n\right]$ be nonnegative definite. The condition (1.2) is equivalent to this condition (see p. 525, [1]). In particular for every $n \times n$ matrix $\left[r\left(t_{k}, t_{l}\right), k, l=1,2, \cdots, n\right]$ to be positive definite it is necessary and sufficient that $u(t)$ and $v(t)$ be positive on $D$ and the strict inequality in (1.2) hold. In connection with our theorems we mention an earlier result by G. Baxter, corollary [1], which showed that if $u_{i}(t)$ and $v_{i}(t)$ have bounded second derivatives on $D=[0,1]$ then for the two subsets $E_{i}, i=1,2$, of $R^{D}$ defined by

$$
\begin{aligned}
E_{i} & =\left\{x \in R^{D} ; \lim _{n \rightarrow \infty} \sum_{k=1}^{2^{n}}\left[x\left(\frac{k}{2^{n}}\right)-x\left(\frac{k-1}{2^{n}}\right)\right]^{2}\right. \\
& \left.=\int_{0}^{1}\left\{u_{i}^{\prime}(t) v_{i}(t)-u_{i}(t) v_{i}^{\prime}(t)\right\} d t\right\}
\end{aligned}
$$

the equalities $\mu_{i}\left(E_{i}\right)=1, i=1,2$, hold so that the condition

$$
\int_{0}^{1}\left\{u_{1}^{\prime}(t) v_{1}(t)-u_{1}(t) v_{1}^{\prime}(t)\right\} d t \neq \int_{0}^{1}\left\{u_{2}^{\prime}(t) v_{2}(t)-u_{2}(t) v_{2}^{\prime}(t)\right\} d t
$$

implies $E_{1} \cap E_{2}=\varnothing$ as well as $\mu_{i}\left(E_{j}\right)=\delta_{i j}$.

The proof of the theorem is given in $\S 3$. For some examples of factorable covariance functions to which our theorem can be applied see J. A. Beekman, pp. 805-806, [2]. 
2. A lemma concerning the inversion of a class of symmetric matrices.

Lemma. Given real or complex numbers

$$
a_{1}, a_{2}, \cdots, a_{n} \quad \text { and } \quad b_{1}, b_{2}, \cdots, b_{n} \text {. }
$$

Let $M=\left[m_{k, l}, k, l=1,2, \cdots, n\right]$ be an $n \times n$ symmetric matrix with entries

$$
m_{k, l}=a_{k} b_{l} \quad \text { for } k \leqq l, \quad k, l=1,2, \cdots, n
$$

Let

$$
\begin{array}{ll}
C_{j}=a_{j} b_{j-1}-a_{j-1} b_{j} & j=2,3, \cdots, n \\
D_{j}=a_{j} b_{j-2}-a_{j-2} b_{j} & j=3,4, \cdots, n
\end{array}
$$

then

$$
\operatorname{det} M=a_{1} b_{n} \prod_{j=2, \cdots, n} C_{j}
$$

For the determinants $M_{k, l}$ of the minor matrices corresponding to the entries $m_{k, l}$ we have

$$
\begin{aligned}
M_{1,1} & =a_{2} b_{n} \prod_{j=3, \cdots, n} C_{j}, & M_{1,2} & =a_{1} b_{n} \prod_{\substack{j=3, \cdots, n \\
M_{1, l}}} C_{j}, \\
M_{1, k} & =0 \text { for } l=3, \cdots, n, & M_{k, k} & =a_{1} b_{n} D_{l+1} \prod_{\substack{k=2, \cdots, n \\
j \neq k, k+1}} C_{j}, \\
M_{k, k+1} & =a_{1} b_{n} \prod_{\substack{j=2, \cdots, n \\
j \neq k+1}} C_{j}, & M_{k, l} & =0 \text { for } l=k+2, \cdots, n,
\end{aligned}
$$

for $k=2, \cdots, n-1$, and finally

$$
M_{n, n}=a_{1} b_{n-1} \prod_{j=2, \cdots, n-1} C_{j} .
$$

In particular $M$ is invertible if and only if $a_{1}, b_{n}, C_{j} \neq 0$ for $j=$ $2, \cdots, n$. In this case

$$
M^{-1}=\left[\frac{(-1)^{k+l}}{\operatorname{det} M} M_{k, l}, k, l=1,2, \cdots, n\right] .
$$

The proof of this lemma is lengthy and will not be given here. We merely mention that the expression (2.1) for $M^{-1}$ can be verified by direct multiplication with $M$.

3. Proof of the theorem. The $J$-divergence of two probability measures $P$ and $Q$ on a measurable space $(\Omega, \mathfrak{B})$ is defined to be 


$$
J(P, Q)= \begin{cases}E_{P}\left[\log \frac{d P}{d Q}(\omega)\right]+E_{Q}\left[\log \frac{d Q}{d P}(\omega)\right] & \begin{array}{l}
\text { when } P \text { and } Q \\
\text { are equivalent } \\
\text { otherwise }
\end{array}\end{cases}
$$

where $E_{P}$ and $E_{Q}$ denote integration with respect to the probability measures $P$ and $Q$.

Let $\mathfrak{F}$ be the smallest $\sigma$-field of subsets of the function space $R^{D}$ with respect to which the real valued function $Y(t, x)=x(t)$ on $R^{D}$ is measurable for every $t \in D$. For $t_{1}, \cdots t_{n} \in D, t_{1}<\cdots<t_{n}$, let

$$
\begin{aligned}
& p_{t_{1} \cdots t_{n}}(x)=\left[x\left(t_{1}\right), \cdots, x\left(t_{n}\right)\right] \quad x \in R^{D} \\
& p_{t_{1} \cdots t_{n}}^{-1}(B)=\left\{x \in R^{D} ;\left[x\left(t_{1}\right), \cdots, x\left(t_{n}\right)\right] \in B\right\} \quad B \in \mathfrak{B}^{n}
\end{aligned}
$$

where $\mathfrak{B}^{n}$ is the $\sigma$-field of Borel sets in the $n$-dimensional Euclidean space $R^{n}$, and let

$$
\mathfrak{F}_{t_{1} \cdots t_{n}}=\left\{p_{t_{1} \cdots t_{n}}^{-1}(B), B \in \mathfrak{B}^{n}\right\} \text {. }
$$

Then $\widetilde{\mho}_{t_{1} \cdots t_{n}}$ is a $\sigma$-field of subsets of $R^{D}$ and $\mho$ is the $\sigma$-field generated by the union of all the $\sigma$-fields $\widetilde{\mho}_{t_{1} \cdots t_{n}}$. Given two probability measures $\mu_{i}, i=1,2$, on $\left(R^{D}, \mathfrak{F}\right)$, let $\mu_{i, t_{1} \cdots t_{n}}=\mu_{i} \mid \mathfrak{F}_{t_{1} \cdots t_{n}}$, i.e., the restrictions of $\mu_{i}$ to the $\sigma$-field $\mathfrak{F}_{t_{1} \cdots t_{n}}$. Let $J=J\left(\mu_{1}, \mu_{2}\right)$ and

$$
J_{t_{1} \cdots t_{n}}=J\left(\mu_{1, t_{1} \cdots t_{n}}, \mu_{2, t_{1} \cdots t_{n}}\right) \text {. }
$$

According to J. Hájek [4], [5], $J=\sup J_{t_{1} \cdots t_{n}}$ where the supremum is taken over the collection of $\left\{t_{1}, \cdots t_{n}\right\}$, i.e., over the collection of all the $\sigma$-fields $\mathfrak{F}_{t_{1} \cdots t_{n}}$, and $J<\infty$ implies the equivalence of $\mu_{1}$ and $\mu_{2}$. Furthermore if $\mu_{1}$ and $\mu_{2}$ are Gaussian then $J=\infty$ implies the singularity of $\mu_{1}$ and $\mu_{2}$.

Let $t_{1}, \cdots, t_{n} \in D, t_{0}<t_{1}<\cdots<t_{n}<t_{0}+\delta$. For the fixed $\left\{t_{1}, \cdots, t_{n}\right\}$ there is a one-to-one correspondence between the members of $\mathfrak{F}_{t_{1} \cdots t_{n}}$ and the members of $\mathfrak{B}^{n}$ according to the definition (3.2). Since the measures $\mu_{i}, i=1,2$, are Gaussian, i.e., the stochastic process $Y(t, x)=x(t)$ is a Gaussian process on each of the two probability spaces $\left(R^{D}, \mathfrak{F}, \mu_{i}\right)$, we have

$$
\mu_{i}\left(p_{t_{1} \cdots t_{n}}^{-1}(B)\right)=\Phi_{i, t_{1} \cdots t_{n}}(B), \quad B \in \mathfrak{B}^{n}, i=1,2
$$

where $\Phi_{i, t_{1} \cdots t_{n}}$ are $n$-dimensional (regular or degenerate) normal distributions on $\left(R^{n}, \mathfrak{B}^{n}\right)$.

Now since $v_{i}(t)>0$ and $u_{i}(t)\left[v_{i}(t)\right]^{-1}$ are strictly increasing on $\left(t_{0}, t_{0}+\delta\right)$ we have

$$
u_{i}\left(t^{\prime \prime}\right) v_{i}\left(t^{\prime}\right)-u_{i}\left(t^{\prime}\right) v_{i}\left(t^{\prime \prime}\right)>0 \text { for } t^{\prime}, t^{\prime \prime} \in\left(t_{0}, t_{0}+\delta\right), t^{\prime}<t^{\prime \prime}, i=1,2 \text {. }
$$

Then the covariance matrices $\left[r_{i}\left(t_{k}, t_{l}\right), k, l=1,2, \cdots, n\right], i=1,2$, of the $n$-dimensional normal distributions $\Phi_{i, t_{1} \cdots t_{n}}$ are positive definite and 
consequently $\Phi_{i t_{1} \cdots t_{n}}$ are regular with density functions given by

$$
\text { (3.4) }=\frac{1}{\left\{(2 \pi)^{n} \operatorname{det} W_{i, t_{1} \cdots t_{n}}\right\}^{1 / 2}} \exp \left\{-\frac{1}{2}\left(W_{i, t_{1} \cdots t_{n}}^{-1} \xi, \xi\right)\right\}, \xi \in R^{n}, i=1,2
$$

where $W_{i, t_{1} \cdots t_{n}}=\left[w_{i, k, l}, k, l=1,2, \cdots, n\right]$ are $n \times n$ symmetric and positive definite matrices with entries

$$
w_{i, k, l}=u_{i}\left(t_{l}\right) v_{i}\left(t_{l}\right) \quad \text { for } \quad k \leqq l, k, l=1,2, \cdots, n, i=1,2 .
$$

Now

$$
\Phi_{i, t_{1} \cdots t_{n}}(B)=\int_{R^{n}} \Phi_{i, t_{1} \cdots t_{n}}^{\prime}(\xi) m_{L}(d \xi), \quad B \in \mathfrak{B}^{n}, i=1,2
$$

where $m_{L}$ is the Lebesgue measure on $\left(R^{n}, \mathfrak{B}^{n}\right)$. The regularity of $\Phi_{1, t_{1} \cdots t_{n}}$ and $\Phi_{2, t_{1} \cdots t_{n}}$ implies their equivalence. This in turn implies the equivalence of $\mu_{1, t_{1} \cdots t_{n}}$ and $\mu_{2, t_{1} \cdots t_{n}}$ on account of the one-to-one correspondence between the members of $\mathfrak{F}_{t_{1} \cdots t_{n}}$ and the members of $\mathfrak{B}^{n}$ and the relation (3.3) between $\mu_{i, t_{1} \cdots t_{n}}$ and $\Phi_{i, t_{1} \cdots t_{n}}$. From (3.6) and (3.4) we obtain the Radon-Nikodym derivatives

$$
\begin{aligned}
& \frac{d \mu_{j, t_{1} \cdots t_{n}}}{d \mu_{i, t_{1} \cdots t_{n}}}(x)=\frac{d \Phi_{j, t_{1} \cdots t_{n}}}{d \Phi_{i, t_{1} \cdots t_{n}}}(\xi)=\frac{\Phi_{j, t_{1} \cdots t_{n}}^{\prime}(\xi)}{\Phi_{i, t_{1} \cdots t_{n}}^{\prime}(\xi)} \\
& \quad=\left[\frac{\operatorname{det} W_{i, t_{1} \cdots t_{n}}}{\operatorname{det} W_{j, t_{1} \cdots t_{n}}}\right]^{1 / 2} \exp \left\{\frac{1}{2}\left(\left[W_{i, t_{1} \cdots t_{n}}^{-1}-W_{j, t_{1} \cdots t_{n}}^{-1}\right] \xi, \xi\right)\right\}, i,=1,2 .
\end{aligned}
$$

According to (3.1), (3.3) and (3.7)

$$
\begin{aligned}
J_{t_{1} \cdots t_{n}} & =E_{\mu_{2, t_{1} \cdots t_{n}}}\left[\log \frac{d \mu_{2, t_{1} \cdots t_{n}}}{d \mu_{1, t_{1} \cdots t_{n}}}(x)\right]+E_{\mu_{1, t_{1}} \cdots t_{n}} \log \left[\frac{d \mu_{1, t_{1} \cdots t_{n}}}{d \mu_{2, t_{1} \cdots t_{n}}}(x)\right] \\
& =E_{\Phi_{2, t_{1} \cdots t_{n}}}\left[\log \frac{\Phi_{2, t_{1} \cdots t_{n}}^{\prime}(\xi)}{\Phi_{1, t_{1} \cdots t_{n}}^{\prime}(\xi)}\right]+E_{\Phi_{2, t_{1} \cdots t_{n}}}\left[\log \frac{\Phi_{1, t_{1} \cdots t_{n}}^{\prime}(\xi)}{\Phi_{2, t_{1} \cdots t_{n}}^{\prime}(\xi)}\right] .
\end{aligned}
$$

In evaluating the integrals in (3.8) we quote the well known equality that for any $n \times n$ matrices $A$ and $B$ where $A$ is symmetric and $B$ is positive definite

$$
\frac{1}{\left.\{2 \pi)^{n} \operatorname{det} B\right\}^{1 / 2}} \int_{R^{n}}(A \xi, \xi) \exp \left\{-\frac{1}{2}\left(B^{-1} \xi, \xi\right)\right\} m_{L}(d \xi)=\operatorname{Tr}(C)
$$

where $C=A B$ and $\operatorname{Tr}(C)=\sum_{k=1}^{n} c_{k, k}$ for $C=\left[c_{k, l}, k, l=1,2, \cdots, n\right]$. Applying (3.9) to (3.8) remembering (3.7), (3.6) and (3.4)

(3.10) $J_{t_{1} \cdots t_{n}}=\frac{1}{2} \operatorname{Tr}\left[W_{1, t_{1} \cdots t_{n}}^{-1} W_{2, t_{1} \cdots t_{n}}+W_{2, t_{1} \cdots t_{n}}^{-1} W_{1, t_{1} \cdots t_{n}}-2 I\right]$.

We proceed to evaluate the diagonal entries of the two product 
matrices in (3.10). Let us consider $W_{1, t_{1} \cdots t_{n}}^{-1} W_{2, t_{1} \cdots t_{n}}$ for example. The entries of $W_{i, t_{1} \cdots t_{n}}$ are given by (3.5). Let $M_{i, k, l}$ be the determinant of the minor matrix corresponding to $w_{i, k, l}$. According to our lemma, $\S 2$, the 1st diagonal entry of $W_{1, t_{1} \cdots t_{n}}^{-1} W_{2, t_{1} \cdots t_{n}}$ is given by

$$
\begin{aligned}
& \frac{M_{1,1,1} w_{2,1,1}-M_{1,1,2} w_{2,1,2}}{\operatorname{det} W_{1, t_{1} \cdots t_{n}}} \\
& =\left[u_{1}\left(t_{2}\right) v_{1}\left(t_{n}\right) u_{2}\left(t_{1}\right) v_{2}\left(t_{1}\right)-u_{1}\left(t_{1}\right) v_{1}\left(t_{n}\right) u_{2}\left(t_{1}\right) v_{2}\left(t_{2}\right)\right] \\
& \quad \cdot\left[u_{1}\left(t_{1}\right) v_{1}\left(t_{n}\right)\left\{u_{1}\left(t_{2}\right) v_{1}\left(t_{1}\right)-u_{1}\left(t_{1}\right) v_{1}\left(t_{2}\right)\right\}\right]^{-1} .
\end{aligned}
$$

The $k$-th diagonal entry, $k \neq 1, n$, is given by

$$
\begin{aligned}
& \frac{-M_{1, k-1, k} w_{2, k-1, k}+M_{1, k, k} w_{2, k, k}-M_{1, k, k+1} w_{2, k, k+1}}{\operatorname{det} W_{1, t_{1} \cdots t_{n}}} \\
& =u_{1}\left(t_{1}\right) v_{1}\left(t_{n}\right)\left[-\left\{u_{1}\left(t_{k+1}\right) v_{1}\left(t_{k}\right)-u_{1}\left(t_{k}\right) v_{1}\left(t_{k+1}\right)\right\} u_{2}\left(t_{k-1}\right) v_{2}\left(t_{k}\right)\right. \\
& \quad+\left\{u_{1}\left(t_{k+1}\right) v_{1}\left(t_{k-1}\right)-u_{1}\left({ }_{k-1}\right) v_{1}\left(t_{k+1}\right)\right\} u_{2}\left(t_{k}\right) v_{2}\left(t_{k}\right) \\
& \quad-\left\{u_{1}\left(t_{k}\right) v_{1}\left(t_{k-1}\right)-u_{1}\left(t_{k-1}\right) v_{1}\left(t_{k}\right)\right\} \\
& \left.\quad \cdot u_{2}\left(t_{k}\right) v_{2}\left(t_{k+1}\right)\right]\left[u _ { 1 } ( t _ { 1 } ) v _ { 1 } ( t _ { n } ) \left\{u_{1}\left(t_{k}\right) v_{1}\left(t_{k-1}\right)\right.\right. \\
& \left.\left.\quad-u_{1}\left(t_{k-1}\right) v_{1}\left(t_{k}\right)\right\}\left\{u_{1}\left(t_{k+1}\right) v_{1}\left(t_{k}\right)-u_{1}\left(t_{k}\right) v_{1}\left(t_{k+1}\right)\right\}\right]^{-1} .
\end{aligned}
$$

Finally, the $n$-th diagonal entry is given by

$$
\begin{aligned}
& \frac{-M_{1, n-1, n} w_{2, n-1, n}+M_{1, n, n} w_{2, n, n}}{\operatorname{det} W_{1, t_{1} \cdots t_{n}}}=\left[-u_{1}\left(t_{1}\right) v_{1}\left(t_{n}\right) u_{2}\left(t_{n-1}\right) v_{2}\left(t_{n}\right)\right. \\
& \left.\quad+u_{1}\left(t_{1}\right) v_{1}\left(t_{n-1}\right) u_{2}\left(t_{n}\right) v_{2}\left(t_{n}\right)\right]\left[u _ { 1 } ( t _ { 1 } ) v _ { 1 } ( t _ { n } ) \left\{u_{1}\left(t_{n}\right) v_{1}\left(t_{n-1}\right)\right.\right. \\
& \left.\left.\quad-u_{1}\left(t_{n-1}\right) v_{1}\left(t_{n}\right)\right\}\right]^{-1}
\end{aligned}
$$

Now according to (1.3)

$$
u_{i}(t)=\lambda_{i}\left(t-t_{0}\right)+\varepsilon_{i}\left(t-t_{0}\right) \quad \text { where } \quad \lim _{t \downarrow t_{0}} \varepsilon_{i}=0, i=1,2 .
$$

For fixed $n$ let $p$ be a sufficiently large positive integer so that $t_{k}=t_{0}+k / p \in\left(t_{0}, t_{0}+\delta\right)$ for $k=1,2, \cdots, n$. Then

$$
\begin{aligned}
u_{i}\left(t_{k}\right)=\frac{k}{p}\left(\lambda_{i}+\varepsilon_{i}\right)= & k \frac{\lambda_{i}}{p}\{1+o(1)\}, \\
& k=1,2, \cdots, n, p \rightarrow \infty, i=1,2 .
\end{aligned}
$$

From (1.4), writing $\nu_{i}$ for $D^{+} v_{i}\left(t_{0}\right)$,

$$
\begin{aligned}
v_{i}\left(t_{k}\right)=r_{i}+k \frac{\nu_{i}}{p}\{1+o(1)\} & =r_{i}\left\{1+O\left(\frac{n}{p}\right)\right\}, \\
k & =1,2, \cdots n, p \rightarrow \infty, i=1,2 .
\end{aligned}
$$

If we apply (3.14) and (3.15) to (3.11), the 1st diagonal entry of $W_{1, t_{1}}^{-1} \therefore t_{n} W_{2, t_{1} \cdots t_{n}}$ is reduced to 


$$
\frac{\lambda_{1} \lambda_{2} r_{1} r_{2} p^{-2}\{2-1\}\{1+o(1)\}\left\{1+O\left(\frac{n}{p}\right)\right\}}{\lambda_{1}^{2} r_{1}^{2} p^{-2}\{2-1\}\{1+o(1)\}\left\{1+O\left(\frac{n}{p}\right)\right\}}=\frac{\lambda_{2} r_{2}}{\lambda_{1} r_{1}}\{1+o(1)\}
$$

Similarly the $k$-th diagonal entry, $k \neq 1, n$, is reduced to

$$
\begin{aligned}
& \frac{\lambda_{1}^{2} \lambda_{2} r_{1}^{2} r_{2} p^{-3}[-\{(k+1)-k\}(k-1)+\{(k+1)-(k-1)\}}{\lambda_{1}^{3} r_{1}^{3} p^{-3}\{k-(k-1)\}\{(k+1)-k\}} \\
& \times \frac{k-\{k-(k-1)\} k]\{1+o(1)\}\left\{1+O\left(\frac{n}{p}\right)\right\}}{\{1+o(1)\}\left\{1+O\left(\frac{n}{p}\right)\right\}}=\frac{\lambda_{2} r_{2}}{\lambda_{1} r_{1}}\{1+o(1)\}
\end{aligned}
$$

and finally the $n$-th diagonal entry is reduced to

$$
\begin{aligned}
\frac{\lambda_{1} \lambda_{2} r_{1} r_{2} p^{-2}\{-(n-1)+n\}\{1+o(1)\}\left\{1+O\left(\frac{n}{p}\right)\right\}}{\lambda_{1}^{2} r_{1}^{2} p^{-2}\{n-(n-1)\}\{1+o(1)\}\left\{1+O\left(\frac{n}{p}\right)\right\}} & \\
& =\frac{\lambda_{2} r_{2}}{\lambda_{1} r_{1}}\{1+o(1)\} .
\end{aligned}
$$

From (3.16), (3.17) and (3.18)

$$
T_{r}\left[W_{1, t_{1} \cdots t_{n}}^{-1} W_{2, t_{1} \cdots t_{n}}\right]=n \frac{\lambda_{2} r_{2}}{\lambda_{1} r_{1}}\{1+o(1)\}, \quad p \rightarrow \infty .
$$

Similarly

$$
T_{r}\left[W _ { 2 , t _ { 1 } \cdots t _ { n } } ^ { - 1 } W _ { 1 , t _ { 1 } \cdots t _ { n } } \left[=n \frac{\lambda_{1} r_{2}}{\lambda_{2} r_{2}}\{1+o(1)\}, \quad p \rightarrow \infty .\right.\right.
$$

Substituting these estimates in (3.10) we obtain

$$
J_{t_{1} \cdots t_{n}}=\frac{n}{2}\left\{\sqrt{\frac{\lambda_{2} r_{2}}{\lambda_{1} r_{1}}}-\sqrt{\frac{\lambda_{1} r_{2}}{\lambda_{2} r_{2}}}\right\}^{2}+n o(1), \quad p \rightarrow \infty .
$$

Since $n$ is fixed, $n o(1) \rightarrow 0$ as $p \rightarrow \infty$. Thus for sufficiently large $p$ chosen for the given $n, n o(1)$ is as small as we wish. Therefore

$$
\sup J_{t_{1} \cdots t_{n}}=\infty \text {. }
$$

This proves the singularity of $\mu_{1}$ and $\mu_{2}$.

\section{BIBLIOGRAPHY}

1. G. Baxter, A strong limit theorem for Gaussian processes, Proc. Amer. Math. Soc. 7 (1956), 522-527. 
2. J. A. Beekman, Gaussian processes and generalized Schroedinger equations, J. Math. Mech. 14 (1965), 789-806.

3. J. Feldman, Equivalence and perpendicularity of Gaussian processes, Pacific J. Math. 8 (1958), 699-708.

4. J. Hájek, A property of J-diveryence of marginal probability distributions, Czechoslovak Math. J. 8 (1958), 460-463.

5. - On a property of normal distributions of an arbitrary stochastic process (in Russian), Czechoslovak Math. J. Vol. 8 (1958), 610-618.

6. A. M. Yaglom, On the equivalence and perpendicularity of two Gaussian probability measures in function space, Proceedings of the Symposium on Time Series Analysis, held at Brown University, 1962, 327-346.

7. J. Yeh, Singularity of Gaussian measures on function spaces induced by Brownian motion processes with non-stationary increments, (to appear shortly in the Illinois J. Math.)

Received April 3, 1969. This research was supported in part by the National Science Foundation Grant NSF GP-8291

UNIVERSity of CALIFORNIA, IRVINe 


\section{PACIFIC JOURNAL OF MATHEMATICS}

\section{EDITORS}

\author{
H. ROYDEN \\ Stanford University \\ Stanford, California \\ Richard Pierce \\ University of Washington \\ Seattle, Washington 98105
}

\author{
J. DugundJI \\ Department of Mathematics \\ University of Southern California \\ Los Angeles, California 90007 \\ BASIL GoRDON \\ University of California \\ Los Angeles, California 90024
}

\section{ASSOCIATE EDITORS}
E. F. BECKENBACH
B. H. Neumann
F. WOLF
K. YoSHIDA

\section{SUPPORTING INSTITUTIONS}

\author{
UNIVERSITY OF BRITISH COLUMBIA \\ CALIFORNIA INSTITUTE OF TECHNOLOGY \\ UNIVERSITY OF CALIFORNIA \\ MONTANA STATE UNIVERSITY \\ UNIVERSITY OF NEVADA \\ NEW MEXICO STATE UNIVERSITY \\ OREGON STATE UNIVERSITY \\ UNIVERSITY OF OREGON \\ OSAKA UNIVERSITY \\ UNIVERSITY OF SOUTHERN CALIFORNIA
}

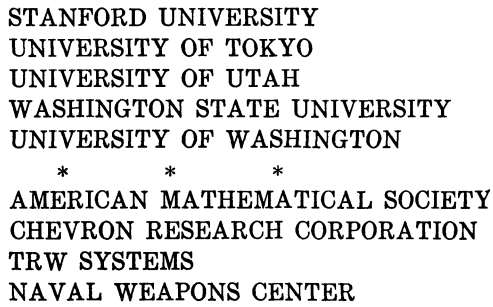

The Supporting Institutions listed above contribute to the cost of publication of this Journal, but they are not owners or publishers and have no responsibility for its content or policies.

Mathematical papers intended for publication in the Pacific Journal of Mathematics should be in typed form or offset-reproduced, double spaced with large margins. Underline Greek letters in red, German in green, and script in blue. The first paragraph or two must be capable of being used separately as a synopsis of the entire paper. It should not contain references to the bibliography. Manuscripts, in duplicate if possible, may be sent to any one of the four editors. Please classify according to the scheme of Math. Rev. 36, 1539-1546. All other communications to the editors should be addressed to the managing editor, Richard Arens, University of California, Los Angeles, California, 90024.

50 reprints are provided free for each article; additional copies may be obtained at cost in multiples of 50 .

The Pacific Journal of Mathematics is published monthly. Effective with Volume 16 the price per volume (3 numbers) is $\$ 8.00$; single issues, $\$ 3.00$. Special price for current issues to individual faculty members of supporting institutions and to individual members of the American Mathematical Society: $\$ 4.00$ per volume; single issues $\$ 1.50$. Back numbers are available.

Subscriptions, orders for back numbers, and changes of address should be sent to Pacific Journal of Mathematics, 103 Highland Boulevard, Berkeley, California, 94708.

PUBLISHED BY PACIFIC JOURNAL OF MATHEMATICS, A NON-PROFIT CORPORATION

Printed at Kokusai Bunken Insatsusha (International Academic Printing Co., Ltd.), 7-17, Fujimi 2-chome, Chiyoda-ku, Tokyo, Japan. 


\section{Pacific Journal of Mathematics}

\section{Vol. 31, No. $2 \quad$ December, 1969}

Efraim Pacillas Armendariz, Quasi-injective modules and stable torsion

classes..........................................

J. Adrian (John) Bondy, On Ulam's conjecture for separable graphs...

Vasily Cateforis and Francis Louis Sandomierski, On commutative rings over which the singular submodule is a direct summand for every module .....

Rafael Van Severen Chacon, Approximation of transformations with continuous

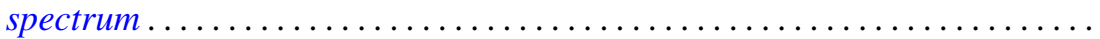

Raymond Frank Dickman and Alan Zame, Functionally compact spaces ...... 303

Ronald George Douglas and Walter Rudin, Approximation by inner

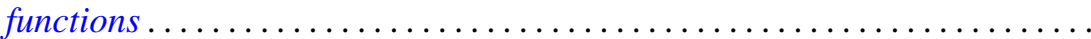

John Walter Duke, A note on the similarity of matrix and its conjugate

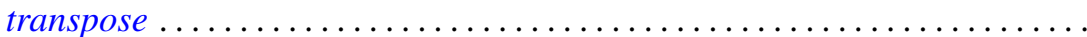

Micheal Neal Dyer and Allan John Sieradski, Coverings of mapping

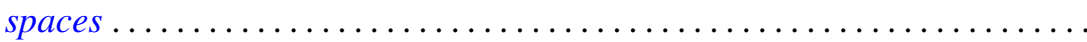

Donald Campbell Dykes, Weakly hypercentral subgroups of finite groups .....

Nancy Dykes, Mappings and realcompact spaces.....................

Edmund H. Feller and Richard Laham Gantos, Completely injective

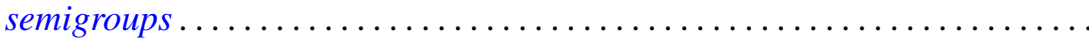

Irving Leonard Glicksberg, Semi-square-summable Fourier-Stieltjes

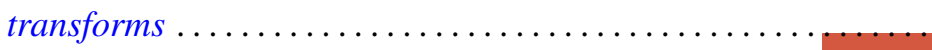

Samuel Irving Goldberg and Kentaro Yano, Integrability of almost cosymplectic structures...

Seymour Haber and Charles Freeman Osgood, On the sum $\sum\langle n \alpha\rangle^{-t}$ and numerical integration ..........................

Sav Roman Harasymiv, Dilations of rapidly decreasing functions ....

William Leonard Harkness and R. Shantaram, Convergence of a sequence of

transformations of distribution functions

Herbert Frederick Kreimer, Jr., A note on the outer Galois theory of rings ...

James Donald Kuelbs, Abstract Wiener spaces and applications to analysis. .

Roland Edwin Larson, Minimal $T_{0}$-spaces and minimal $T_{D}$-spaces...

A. Meir and Ambikeshwar Sharma, On Ilyeff's conjecture .

Isaac Namioka and Robert Ralph Phelps, Tensor products of compact convex sets....

James L. Rovnyak, On the theory of unbounded Toeplitz operators ....

Benjamin L. Schwartz, Infinite self-interchange graphs.......

George Szeto, On the Brauer splitting theorem...

Takayuki Tamura, Semigroups satisfying identity $x y=f(x$,

Kenneth Tolo, Factorizable semigroups .................. 